\title{
Physiological and Ethological Impacts of the Antidepressant Escitalopram Studied on Ants as Models
}

\section{Marie-Claire Cammaerts*}

Independent Researcher, Retired from the Biology of Organisms Department, University of Brussels, Belgium

*Corresponding Author: Marie-Claire Cammaerts, Independent Researcher, Retired from the Biology of Organisms Department, University of Brussels, Belgium.
Received: April 05, 2021

Published: May 06, 2021

(C) All rights are reserved by Marie-Claire

Cammaerts.

\begin{abstract}
Humans' consumption of antidepressants is nowadays increasing. One of the most used for treating severe depressions is escitalopram, an inhibitor of the serotonin recapture, and being so, even if efficient, it may have harmful side effects. Using ants as models, we studied the effect of escitalopram on 13 physiological and ethological traits, the potential adaptation to these effects, the dependence on escitalopram consumption, and the loss of the effect of the drug after weaning. We found that escitalopram impacted the ants' food consumption, general activity, orientation ability, tactile perception, social relationships, cognition, learning and memory. The ants did not adapt themselves to the impact of escitalopram and did not develop dependence on this drug consumption. Young ants seemed to be more affected than the old ones by escitalopram consumption. After weaning, the effect of escitalopram first remained intact during 6 hours, and then slowly decreased in about 30 hours, what accounted for the no dependence on this drug consumption. Most of our results agree with effects observed in humans and with the dosage advised to patients (a dose of 10 mg every 24 hours). A few side effects seen in ants have not (or not yet) been observed in humans and should be thus examined (e.g. effect on social relationships, learning and memory). Habituation to the positive effect of escitalopram, impossible to study on ants, should be checked in humans since in case of its occurrence, humans will increase their drug consumption and therefore the impact of the side effects.
\end{abstract}

Keywords: Cognition; Food Intake; Learning; Memory; Myrmica sabuleti

\section{Abbreviations}

ang.deg.: Angular Degrees; ang.deg./cm: Angular Degrees Per $\mathrm{cm} ; \mathrm{mm} / \mathrm{s}$ : Millimeter Per Second; $\chi^{2}$ : Chi-Square; vs: Versus; ${ }^{\circ}$ : Number; cm: Centimeter; mm: Millimeter; ml: Milliliter; mg: Milligram; s: Second; min: Minute; h: Hour; t: Time; \%: Percentage

\section{Introduction}

Together with antibiotics, analgesics, anxiolytics and hormones, the antidepressants are among the most consumed drugs in the world [1]. Due to the economical, social and psychological troubles generated by the long lasting covid-19 pandemic, their use is nowadays increasing [2]. Several kinds of antidepressants exist. The ISSR (the inhibitors of the serotonin recapture) have for instance fluoxetine or paroxetine as active substance; such drugs are for examples Prosac, Luvox. The ISNaR (the inhibitors of the serotonin and noradrenalin recapture) have for instance venlafaxine as active substance; such drugs are for example Efexor, Savella. The ATC drugs (the tricyclic antidepressants) inhibit several neurotransmitters and have for instance clomipramine hydrochloride or amitriptyline as active substance; examples of such drugs are Anafranil and Redomex. We have studied physiological and ethological impacts of 
fluoxetine, paroxetine, venlafaxine, clomipramine hydrochloride and amitriptyline on ants used as biological models [3-6]. Fluoxetine was found to be the more toxic drug even leading to death. Paroxetine as well as venlafaxine appeared to be somewhat less toxic. Clomipramine hydrochloride and amitriptyline presented the less severe adverse effects. However, they are not sufficient for caring of patients suffering from pronounced depressions, and such patients become actually more and more numerous due to mental impairments caused by the covid-19 pandemic. A strong nowadays largely used antidepressant is escitalopram (Dr Van Laer, Bruxelles, Belgium, personal communication). Being an ISRS (as fluoxetine), it may present harmful side effects maybe not divulgated or not researched. We thus intended to examine the impacts of escitalopram on several ants' physiological and ethological traits in the same way we did for the other previously studied antidepressants. Hereunder, we report available information on escitalopram, explain why using ants as models is possible and advantageous, say which species we used and what we know on it, and which physiological and ethological traits we aimed to consider.

\section{Information available on escitalopram}

The drug citalopran contains the S-enantiomer escitalopram and the R-enantiomer citalopram. In vivo and in vitro pharmacological studies have proved that escitalopram, which selectively inhibits the serotonine recapture, is more efficient than the citalopram and the R-citalopram, may be the most nowadays selective ISRS, and has rather weak affinity for the histaminergic, muscarinic and adrenergic neurotransmitters [7-9]. It may thus have less adverse effects than the other ISRS. Escitalopram has also been shown to be efficient for treating patients suffering from severe depression [10-12]. Though several medicinal works affirm that escitalopram have very few adverse effects and is well tolerated by the patients $[13,14]$, the notice for use joined to this drug package reports a lot of possible health impairments due to escitalopram consumption. The most common of these impairments are nausea, headache, decrease or increase of food intake, muscular pain, tiredness, weight gain or loss, nervousness, visual perception problems, aggressiveness, edema, fever, confusion, suicidal thoughts, akathisia. However, these harmful side effects seem to be lower and better tolerated by patients than those caused by other antidepressants since the patients treated with escitalopram less often interrupt their treatment [15]. Most patients having had an AVC suffer from a severe depression. Treated with escitalopram, they feel largely better than cared of thanks to psychotherapy, and they easily support the drug side effects [16]. Depressions occurring in more than 65-year old persons severely imperil their survival. Limited studies have shown that, treated with citalopram, these old patients seem less depressed and present few unwanted side effects [17]. A study on maternally separated mouse pups revealed the supremacy of escitalopram over other antidepressants as for its efficiency in reducing anxiousness and its tolerability [18]. A clinical study on depressed patients has shown that escitalopram was efficient in ameliorating their health while presenting few adverse effects, the latter being nausea, insomnia, ejaculation disorder, diarrhea, dry mouth and somnolence [19]. A detailed analysis of escitalopram side effects and their consequences on the patients' use of the drug has proved that these side effects did not statistically incite the patients to reduce or interrupt their treatment [20]. Even if many investigation on the potential adverse effects of escitalopram have been made, nothing is until now reported about the impact of this drug on patients' locomotion, orientation ability, audacity, sensory perception, social relationships, cognition, learning, memory, adaptation to side effects, habituation to wanted effects, dependence on the drug consumption, loss of its effects after its consumption was stopped. We intended to fill this gap working as previously on ants used as models [21]. Hereunder, we recall why using ants as models, which species we used and what we know on it, then the physiological and ethological traits which may be affected by escitalopram we aimed to examine.

\section{Reasons for using ants as models}

Most biological processes are similar in all animals including humans (muscles contraction, nervous influx, sensory perception, digestion, memorization...). Several invertebrates and vertebrates are therefore used as biological models [22,23]. Invertebrates are preferentially used due to their small size, easy maintenance, and short life cycle [24]. Insects are often used, e.g. the fruit flies, the bees [25]. Ants can thus also be used as models. They can advantageously serve as biological models for two reasons. First, their maintenance in a laboratory is very easy and not at all expensive. Secondly, they present many evolved biological characters on which impacts of products or situations can be examined. Among others, 
they can memorized cues and use them to navigate, they use specific chemical substances (pheromones) for informing nestmates, collecting food, recruiting nestmates, adequately marking different areas of their environment. They also take care of their brood, build complex nests, clean them and manage cemeteries at the frontiers of their territory. They possess many cognitive abilities allowing them performing rather complex tasks, and the impact of products on such performances can be examined [26].

The species we used, what we know on it

We use the species Myrmica sabuleti Meinerts 1861. We know its recruitment system, navigation strategy, visual perception, visual and olfactory conditioning [27], and the ontogenesis of several of their abilities [28]. They can recognize themselves in a mirror, become imprinted at their emergence to the front head of their congeners, and learn the alarm and the trail signals in the presence of older congeners $[29,30]$. They natively possess a number line, acquire the notion of zero through experiences, and can acquire and use numerical symbolisms [30,31]. They are submitted to the distance effect, the size effect and the Weber's law [32,33]. They can fore think the time and the location of the next food delivery $[34,35]$, as well as the future amount of elements present aside their food [36]. Nevertheless, they cannot use sensu stricto tools [37]. In fact, their cognitive abilities stay at a concrete level and never reach abstraction.

\section{The traits we examined}

We examined the impact of escitalopram on the ants' food consumption, general activity, locomotion, orientation ability, audacity, tactile perception, social relationships, cognition, stress, learning and memory, as well as their adaptation to an adverse effect of the drug, their habituation to a positive effect of the drug, their dependence on its consumption, and the decrease of its effect after weaning. Our experimental protocols being identical to previous ones [38-40], we here rather briefly explain them, though not avoiding inevitable plagiarism. We affirm having made our entire experimental work being blind to the actual knowledge on escitalopram side effects.

\section{Materials and Methods}

\section{Collection and maintenance of ants}

The experiments were made on two colonies of $M$. sabuleti collected in September 2019 in an abandoned quarry located at Olloy-sur-Viroin (Belgium, 50 04' north, $4^{\circ} 36^{\prime}$ east). Each colo- ny contained about 500 workers, a queen and brood. They were maintained in one to three glass tubes, half-filled with water, a cotton plug separating the ants from the water. The nest tubes of each colony were deposited in a tray $(34 \mathrm{~cm} \times 23 \mathrm{~cm} \times 4 \mathrm{~cm}$ ) serving as foraging area. In the latter area, a cotton-plugged tube filled with an aqueous solution of sugar was permanently provided, and pieces of Tenebrio molitor larvae (Linnaeus, 1758) were delivered three times per week. The lighting equaled ca 330 lux, the temperature $c a 20^{\circ} \mathrm{C}$, the humidity $c a 80 \%$, and the electromagnetic field $c a$ $2 \mu \mathrm{Wm}^{2}$. These conditions were suitable to the used species. The word 'ants' is here often replaced by 'workers' or 'nestmates' as commonly done by researchers on social insects.

\section{Solution of escitalopram given to the ants}

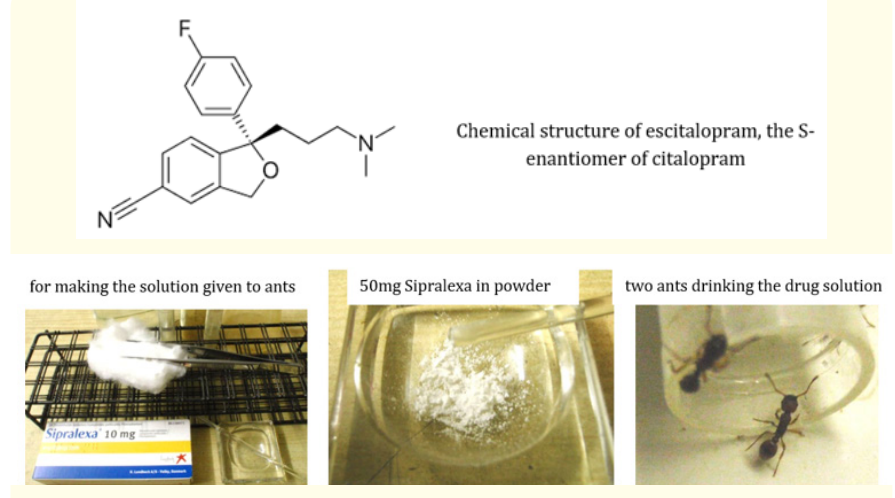

Figure 1: Realisation of the sugared solution of escitalopram given to the ants.

A package of tablets of Sipralexa ${ }^{\circledR} 10 \mathrm{mg}$ (BE238971), (produced by H. Lundbeck A/S, Ottiliavej 9, 2500 Valby, Danemark; authorized by Lundbeck s.a., Stephanie Square Centre - Avenue Louise $65 / 11$ - 1050 Bruxelles, Belgium), was furnished by the pharmacist Wera (Brussels, Belgium). Each tablet contained 10mg of the active substance escitalopram. Human adults treated with this drug are advised to consume one tablet (10mg of escitalopram) per day. As mammals, humans consume about one liter of water per day. Due to their anatomy and morphology, the insects, and thus ants, consume about ten times less water than mammals. Therefore, to maintain ants under an escitalopram diet similar to that of humans, they must receive a solution of $10 \mathrm{mg}$ of escitalopram, that is of one tablet of Sipralexa ${ }^{\circledR}$, in $100 \mathrm{ml}$ of water, or of $5 \mathrm{mg}$ of escitalopram, that is a half tablet of Sipralexa ${ }^{\circledR}$, in $50 \mathrm{ml}$ of water. A solution of a half tablet of Sipralexa ${ }^{\circledR}$ containing $5 \mathrm{mg}$ of escitalopram was thus realized (Figure 1). The half tablet was crushed then dissolved into 
$50 \mathrm{ml}$ of sugared water, and the obtained solution was delivered to the ants in their usual cotton-plugged tubes. The plug of these tubes was refreshed every 2 - 3 days, and the entire solution was renewed every 7 days. Several times per day, we checked if ants drunk the provided solution and they did though only little. The control experiments were firstly made on the colonies maintained under normal diet. Then, their tube containing sugared water was replaced by a tube contained the aqueous sugared solution of excitalopram, and the test experiments were made on these colonies. The latter experiments started after the ants had the drug solution at their disposal during one day.

\section{Assessment of food consumption and general activity}

For ants under normal diet then for them under a diet with escitalopram, those present on the meat food, at the entrance of the sugar water tube, and being active at any place (in the foraging area, at the nest entrance, inside the nest) were separately counted. These punctual counts were made four times during the day and two times during the night (total $=6$ counts $\times 2$ colonies $=12$ counts per day) during 6 days, each day at the same time o'clock. For each kind of diet and of trait, the daily mean was established (Table 1, lines 1 - 6). The mean of the six daily means was then calculated for each kind of diet and of trait (Table 1, last line). For each kind of trait (meat intake, sugar water intake, general activity), the six daily means obtained for ants consuming escitalopram were compared to the six daily means obtained for ants under normal diet using the non-parametric test of Wilcoxon, the level of significance being 0.05 [41].

Assessment of the ants' linear speed, angular speed and orientation ability

These assessments were performed on ants freely moving in their foraging area, those of linear and angular speeds without stimulating the ants, that of orientation while stimulating them with a nestmate tied to a piece of paper (Figure 2A). A tied nestmate emits its attractive mandible glands alarm pheromone, what attractive surrounding ants. To assess the ants' speeds as well as their orientation, 40 trajectories were recorded and analyzed using appropriate software set up according to the following definitions [42]. The linear speed (in millimeter per second $=\mathrm{mm} / \mathrm{s}$ ) is the length of a trajectory divided by the time spent to travel it; the angular speed (in angular degrees per centimeter = ang.deg./cm) is the sum of the angles made by successive adjacent segments, divided by the length of the trajectory; the orientation (in angular degrees = ang. deg.) to a location is the sum of successive angles made by the direction to the location and the direction of the trajec- tory, divided by the number of measured angles. A value lower than $90^{\circ}$ means that the animal has a tendency to orient itself toward the location; a value higher than $90^{\circ}$ signifies that it tends to avoid the location. For each three variables (linear speed, angular speed, orientation), the median and quartiles of the 40 obtained values were established (Table 2, lines 1, 2, 3). The distributions obtained for ants consuming escitalopram were compared to those obtained for ants under normal diet using the non-parametric $\chi^{2}$ test [41].

\section{Assessment of the ants' audacity}

A cylindrical tower (height $=4 \mathrm{~cm}$; diameter $=1.5 \mathrm{~cm}$ ) tied to a squared platform $\left(9 \mathrm{~cm}^{2}\right)$, both in Steinbach ${ }^{\circledR}$ white paper, was deposited in the ants' foraging area. Then, the ants present on this apparatus were counted 10 times over 10 minutes (Figure 2B). The mean and the extremes of the recorded numbers were established (Table 2, line 4). The numbers obtained for the two colonies were added and those relative to every two successive minutes were added, what provided five successive sums. The latter sums obtained for ants under escitalopram diet were compared to those obtained for ants under normal diet using the non-parametric test of Wilcoxon [41].

\section{Assessment of the ants' tactile (pain) perception}

Ants duly perceiving the uncomfortable character of a rough substrate walk there with difficulty, slowly, sinuously, often touching the substrate with their antennae (Figure 2C1). Ants poorly perceiving the rough uncomfortable character of a substrate walk there abnormally more easily, more quickly and less sinuously. Therefore, to evaluate the ants' tactile (probably pain) perception, we assessed their linear and angular speeds while they walked on a rough substrate. A piece $(3 \mathrm{~cm} \times 2+7+2=11 \mathrm{~cm})$ of $\mathrm{n}^{\circ} 280$ emery paper was duly folded and set in a tray $(15 \mathrm{~cm} \times 7 \mathrm{~cm} \times$ $4.5 \mathrm{~cm}$ ) dividing thus this tray in a $3 \mathrm{~cm}$ long zone, a $3 \mathrm{~cm}$ long one covered with the emery paper, and a $9 \mathrm{~cm}$ long zone. For making an experiment on a colony, 12 ants were deposited in the first free of emery paper $3 \mathrm{~cm}$ long zone of the apparatus, and their trajectories were recorded when they tried to cross the zone containing the emery paper. Their linear and angular speeds were quantified as explained in the subsection relative to linear and angular speeds. Two colonies having been experimented, 24 values of linear and angular speeds were recorded, and for each kind of speed, the median and quartiles of the obtained values were established (Table 2, lines 5, 6). The distributions relative to ants consuming escitalopram were compared to those relative to ants under normal diet using the non-parametric $\chi^{2}$ test [41]. 


\section{Assessment of the ants' brood caring behavior}

For each colony, a few larvae were removed from the nest and deposited in front of the nest entrance. Five of them were observed during five minutes (total number of observed larvae $=10$ ). We observed only five larvae for each colony because we must track all of them simultaneously during 5 minutes. The experiment was not repeated because it causes a harmful perturbation to the colony. We cautiously observed the ants' behavior in front of these $2 \times 5$ tracked larvae (Figure 2D), and counted those not re-entered in the nest after 30 seconds, 1, 2, 3, 4 and 5 minutes. The six numbers obtained for the two colonies were correspondingly added (Table 3 , line 1). The six sums obtained for ants consuming escitalopram were compared to the six sums obtained for ants under normal diet using the non-parametric test of Wilcoxon [41].

\section{Assessment of ants' social relationships}

Nestmates normally behave peacefully, are not aggressive towards one another. For examining the potential effect of escitalopram on the nestmates' peaceful interactions, five ants' dyadic encounters were conducted for each colony (total of encounters $=10$ ). Each encounter was performed in a cup (diameter $=2 \mathrm{~cm}$, height $=1.6 \mathrm{~cm}$ ) the borders of which having been lightly covered with talc to prevent the ants from escaping. During each encounter, one ant of the pair was cautiously observed during 5 minute. Its behavior was characterized by the numbers of times it did nothing (level 0 of aggressiveness), touched the other ant with its antennae (level 1), opened its mandibles (level 2), gripped and/or pulled the other ant (level 3), and tried to sting or stung the other ant (level 4) (Figure 2E). The corresponding numbers obtained for each ants and each colony were added what provided a distribution of values (Table 3, line 2). The distribution obtained for ants consuming escitalopram were compared to that obtained for ants under normal diet using the non-parametric $\chi^{2}$ test [41]. For each kind of diet, the ants' social relationship was also assessed by a variable ' $a$ ' equaled to the number of aggressiveness levels $2+3+4$ divided by the number of aggressive levels $0+1$ (Table 3 , line 2 ).

Assessment of ants' cognition (ability of finding their way in a maze)

This assessment was made in tray $(15 \mathrm{~cm} \times 7 \mathrm{~cm} \times 4.5 \mathrm{~cm})$ containing two pieces of paper (Steinbach ${ }^{\circledR}, 12 \mathrm{~cm} \times 4.5 \mathrm{~cm}$ ) duly folded for creating a twists and turns path between a $2 \mathrm{~cm}$ long area in front of this "difficult" path and a $8 \mathrm{~cm}$ long area beyond that path. Each colony had its own device. For each colony, 15 ants were transported into the area lying in front of the twists and turns path (Figure 3A). Since that time, the ants still in front of the difficult path and those having reached the area lying beyond it were counted after 2, 4, 6, 8, 10 and 12 minutes. The corresponding numbers obtained for the two colonies were added (Table 3, line 3 ). The numbers obtained for each of the two considered areas relative to ants consuming escitalopram were compared to those obtained for ants under normal diet using the non-parametric Wilcoxon test [41].

\section{Assessment of the ants' cognition and stress}

Escaping from an enclosure requires being calm, looking calmly to an exit and presenting some cognitive ability. For assessing these traits, six ants of each colony were enclosed under a reversed polyacetate cup (height $=8 \mathrm{~cm}$, bottom diameter $=7 \mathrm{~cm}$, ceiling diameter $=5 \mathrm{~cm}$, the inside surface being slightly covered with talc) deposited in their foraging area. A notch ( $3 \mathrm{~mm}$ height, $2 \mathrm{~mm}$ broad) had been managed in the rim of the bottom of the cup through which the ants could escape (Figure $2 \mathrm{~F}$ ). The enclosed ants of each colony escaped after $2,4,6,8,10$ and 12 minutes were counted and the corresponding numbers obtained for the two colonies were added (Table 3, line 4). The six sums obtained for ants consuming escitalopram were compared to those obtained for ants under normal diet using the non-parametric Wilcoxon test [41].

Assessment of the ants' conditioning acquisition, and memory

For each colony provided with a solution of escitalopram, at a given time, a yellow hollow cube made in strong paper (Canson ${ }^{\circledR}$ ) was deposited above the entrance of the tube containing that solution and the pieces of mealworms were set near that tube. The ants underwent so operant visual conditioning. The control experiment on ants under normal diet had been previously made on another similar colony since when an individual has acquired conditioning to a stimulus, it kept its conditioning during two to three days and even after it has lost it, it more quickly than usually acquires it again. It can thus no longer be used for again assessing its conditioning acquisition. The results of this previously made control experiment can be found in a published paper [43]. Over the ants' conditioning acquisition, then after the yellow cube removal, over the ants' loss of conditioning, the ants were tested in an own Y-apparatus made of strong white paper, its sides having been slightly covered with talc, and deposited in a separated tray. Each Y-apparatus was provided with a yellow hollow cube in one of its branch; half of the tests were made with the cube in the left 
branch and the other half with the cube in the right branch of the Y-maze. To make a test on a colony, 10 ants were transferred one by one in the area lying in front of the two branches of the Y-maze. The ants' first choice of one or the other branch of the Y-apparatus was recorded (Figure 3B). Choosing the branch containing the yellow cube was considered as giving the correct response. Each tested ant was momentarily deposited into a cup until 10 ants of its colony were tested. After the test, the 10 ants were transferred back into their foraging area. The responses given by the ants of the two colonies were added ( $\mathrm{n}=10 \times 2=20$ responses) and the proportions of correct responses (= the conditioning scores) obtained over time were calculated (Table 4). The numbers of correct responses obtained over time for ants consuming escitalopram were compared to those previously obtained for ants living under normal diet using the non-parametric Wilcoxon test [41].

Study of ants' potential adaptation to an adverse effect of escitalopram

An individual becomes adapted to a drug or a situation when it less and less suffers from the adverse effects of this drug or situation over time. To study adaptation, a trait affected by the drug or the situation must be examined after one or two days of their use, then again after several days of their use and the results of the two tests must be compared. In the present work, a trait largely impacted by escitalopram was the ants' orientation ability. Therefore, this trait was again assessed after the ants consumed the drug during eight days exactly as it had been assessed after two days of consumption. The median and quartiles of the obtained values were established (Table 5, upper part), and the distributions of the obtained values were compared to those obtained after three days of escitalopram consumption as well as to those obtained for ants living under normal diet using the non-parametric $\chi^{2}$ test [41].

Study of ants' potential habituation to a positive effect of escitalopram

An individual becomes habituated to a drug or a situation when it becomes over time less and less sensitive to the expected effect of the drug or the situation. To study habituation, a trait favorably affected by the drug or the situation must be examined one or two days after the drug or the situation use and then again after their use during several days, and the results of the two assessments must be compared. In the present work, no positive effect of escitalopram could be pointed out. Habituation was thus not here studied. However, it may occur in humans, what may lead them to increase their consumed dose. Habituation should thus be examined in humans consuming this drug.

Assessment of the ants' dependence on escitalopram consumption

An individual acquires dependence on a drug or a situation when it enjoys using this drug or situation, tries to have them at its disposal, goes on using them whatever their side effects and finally can no longer live without them. In the present work, the ants' dependence on escitalopram was studied after the ants had consumed this drug for 10 days. To do so, 15 ants of each colony were transferred into an own tray $(15 \mathrm{~cm} \times 7 \mathrm{~cm} \times 5 \mathrm{~cm})$ which contained two cotton-plugged tubes $(\mathrm{h}=2.5 \mathrm{~cm}$, diam. $=0.5 \mathrm{~cm})$, one filled with sugar water, the other with the sugared solution of escitalopram used all over the experimental work. The tube containing the drug was located on the right in one tray and on the left in the other tray (Figure 3C). For each colony, one minute after the ants' deposit, those present at the entrance of each tube were counted 15 times over 15 minutes, and the 15 counts obtained for the two colonies were correspondingly added (Table 5, lower part). The two obtained totals allowed calculating the proportion of the ants' choice of the drug-free solution on one hand and of the drug solution on the other hand. Also, the two obtained totals were compared to the two numbers expected if the ants randomly went onto the two presented tubes, using the non-parametric $\chi^{2}$ goodness-offit test [41].

Study of the decrease of the effect of escitalopram after its consumption was stopped

This decrease was studied after the ants had consumed escitalopram during 14 days, and the study was based on the effect of the drug on the ants' orientation ability. The ants were provided with a fresh solution of escitalopram 12 hours before the start of the study. At the end of these 12 hours, the ants' orientation ability was assessed (at $\mathrm{t}=0$ ) just like it had been after three and eight days of the drug consumption, except that 20 instead of 40 ants' trajectories were recorded and analyzed in order to succeed in finalizing the successive orientation assessments all along the decrease of the effect of escitalopram on this trait. After this assessment, the tubes filled with the sugared solution of escitalopram were removed and replaced by tubes filled of the usual aqueous sugar solution. Since this time, weaning started, and the ants' orientation ability was assessed every three hours until it became similar to that of ants living under normal diet, i.e. to the control. For each assessment, the median and quartiles of the 20 obtained values were 
calculated (Table 6). Also, for each assessment, the distribution of the obtained values was compared to that obtained at $\mathrm{t}=0$ and to the control one using the non-parametric $\chi^{2}$ test (Table 6) [41]. The results are also illustrated in figure 4 . The mathematical function describing the best the increase of orientation ability (the decrease of escatalopram effect) over time after weaning was approximately established and is reported in the results section.

\begin{tabular}{|c|c|c|c|c|c|c|}
\hline Days & \multicolumn{3}{|c|}{ Normal diet } & \multicolumn{3}{c|}{ Diet with escitalopram } \\
\hline & Meat & Sugar water & Activity & Meat & Sugar water & Activity \\
\hline I & 1.08 & 1.75 & 6.50 & 0.50 & 0.50 & 5.75 \\
\hline II & 1.50 & 1.50 & 7.50 & 0.50 & 0.25 & 5.75 \\
\hline III & 1.50 & 1.25 & 7.25 & 0.75 & 0.25 & 6.00 \\
\hline IV & 1.00 & 1.50 & 7.50 & 0.25 & 0.50 & 6.75 \\
\hline V & 1.25 & 1.50 & 11.00 & 0.75 & 0.50 & 5.00 \\
\hline VI & 1.50 & 1.75 & 10.00 & 0.75 & 0.50 & 5.00 \\
\hline I-VI & 1.31 & 1.54 & 8.29 & 0.58 & 0.42 & 5.71 \\
\hline
\end{tabular}

Table 1: Impact of escitalopram on the ants' food intake and general activity.

The table gives, for six successive days, the mean number of ants sighted on their meat, on their sugar water, and being active anywhere, as well as, for each kind of counts, the mean of the six daily means. Escitalopram significantly decreased all these three examined traits. Details are given in the text.

\begin{tabular}{|c|c|c|}
\hline Traits & Normal diet & Diet with escitalopram \\
\hline Linear speed (mm/s) & $10.5(9.6-11.3)$ & $8.9(8.2-9.8)$ \\
\hline $\begin{array}{c}\text { Angular speed } \\
\text { (ang.deg./cm) }\end{array}$ & $115(102-126)$ & $176(162-207)$ \\
\hline Orientation (ang.deg.) & $28.4(24.8-40.2)$ & $62.6(51.9-81.1)$ \\
\hline Audacity ( $\left.{ }^{\circ}\right)$ & $2.85[2-4]$ & $1.60[1-3]$ \\
\hline $\begin{array}{c}\text { Linear speed (mm/s) } \\
\text { Angular speed } \\
\text { (ang.deg./cm) } \\
\text { on a rough substrate }\end{array}$ & $280(252-301)$ & $173(157-200)$ \\
\hline
\end{tabular}

Table 2: Impact of escitalopram on five ants' ethological and physiological traits.

The table gives the median (and the quartiles) or the mean [and the extremes] of the recorded values. Escitalopram affected each four examined traits. Details and statistics can be found in the text.

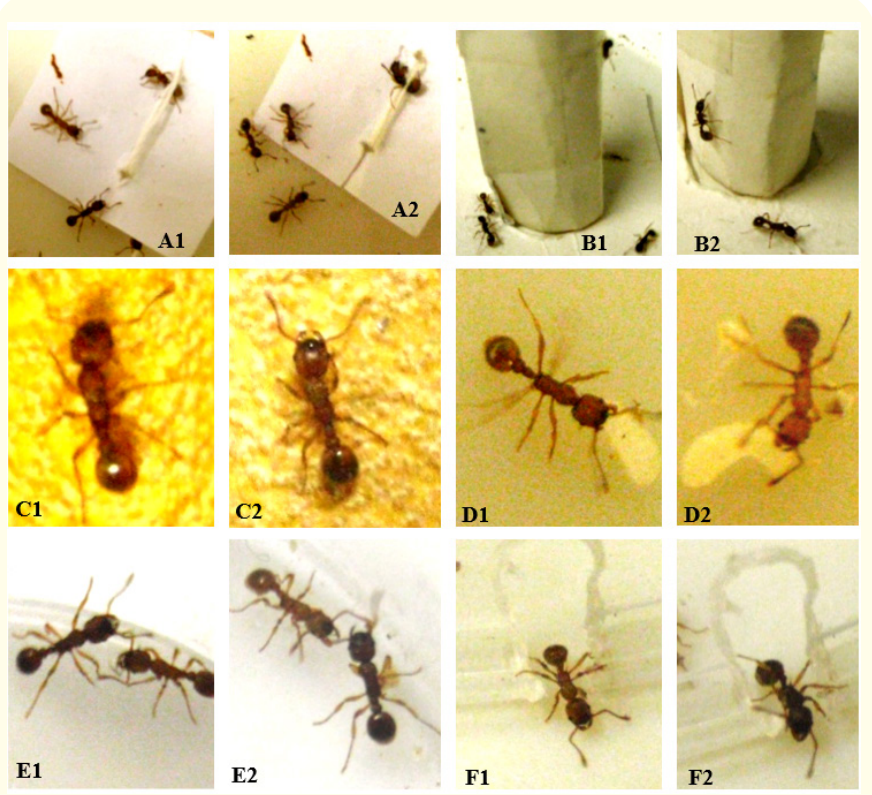

Figure 2: Ant(s) 1 under normal; 2 under escitalopram diet. A: coming to a tied nestmate, less well when under the drug diet. B: coming onto an unknown apparatus, less numerously while consuming escitalopram. C: walking on a rough substrate, less easily and touching the substrate with its antennae while under normal diet. D: under normal diet holding a larva, consuming escitalopram having difficulties to do so. E: staying peaceful aside each other while under normal diet, being somewhat aggressive (opening their mandibles) while consuming escitalopram. F: under normal diet easily escaping, consuming the drug doing so with difficulty.

\section{Results}

Food consumption, general activity

Escitalopram affected these three ants' physiological traits (Table 1). While consuming this drug, the ants eat less meat, drunk less sugar water, and were less active than while living under normal diet and this was significant (for each kind of trait: $\mathrm{N}=6, \mathrm{~T}=-21, \mathrm{P}$ $=0.016$ ). Such an effect of the drug was obvious to the observers. For example, very often, only one ant was walking all around the meat food without coming onto it and eating it.

\section{Linear and angular speeds}

Escitalopram consumption impacted the ants' locomotion. Under that drug diet, the ants walked more slowly and more sinuously than when living under normal diet (Table 2, lines 1,2). These differences were statistically significant: linear speed: $\chi^{2}=11.79, \mathrm{df}$ $=2,0.001<\mathrm{P}<0.01$; angular speed: $\chi^{2}=52.60, \mathrm{df}=2, \mathrm{P}<0.001$. 
Young ants, a few months old, with a clear cuticle, seemed to be more affected than the old 2 to 3 years old ants. Their linear speed meanly equaled $4.2 \mathrm{~mm} / \mathrm{sec}$ and their angular speed 328 ang.deg./ $\mathrm{cm}$. Such a presumed difference between young and old ants as for the impact of escitalopram on their physiology and/or ethology was attentively observed in the course of the experiments for which young ants could be used, i.e. the experiments relative to tactile (pain) perception, brood caring and dependence on the drug consumption.

\section{Orientation ability}

Escitalopram affected this ants' trait. Ants under normal diet rapidly correctly oriented themselves towards a tied nestmate. While consuming escitalopram, they poorly did so (Table 2, line 3; Figure $2 \mathrm{~A}$ ). The difference of orientation ability between the ants under one and the other kinds of diet was significant: $\chi^{2}=44.37$, $\mathrm{df}=2, \mathrm{P}<0.001$. Young ants could not be here experimented since they did not yet know their species alarm pheromone and reaction and will learn them only later, when being older, in the presence of nestmates presenting the alarm reaction [44].

\section{Audacity}

While consuming escitalopram, fewer ants came onto the presented unknown risky apparatus and stayed on it during less time than when living under normal diet (Table 2, line 4; Figure 2 B). This difference was significant $(\mathrm{N}=5, \mathrm{~T}=-15, \mathrm{P}=0.031)$ and may be due to the ants' lower activity while consuming escitalopram (see the here above subsection relative to general activity). It was also observed that the ants coming onto the apparatus often opened their mandibles, and that those climbing on the tower rather often fell from it. No young ant came onto the apparatus, what was usual.

\section{Tactile (pain) perception}

Escitalopram decreased the ants' tactile perception (Table 2, lines 5, 6; Figure 2C). While consuming this drug, the ants walked on a rough substrate more quickly, less sinuously and more frankly than when living under normal diet. Also, they far less often touched the substrate with their antennae. The difference of locomotion on a rough substrate of ants under one and the other kinds of diet was significant: linear speed: $\chi^{2}=40.80, \mathrm{df}=2, \mathrm{P}<$ 0.001; angular speed: $\chi^{2}=27.77, \mathrm{df}=1, \mathrm{P}<0.001$. Escitalopram may thus reduce the ants' sensory perception, a presumption in agreement with their poor ability in orienting themselves towards a source of alarm pheromone (see the above subsection relative to the ants' orientation ability). A few young ants were here cautiously observed: they obviously walked nearly like as being on a usual substrate, very poorly perceiving the rough character of the emery paper.

\begin{tabular}{|c|c|c|}
\hline Traits & Normal diet & Diet with escitalopram \\
\hline $\begin{array}{l}\text { Brood } \\
\text { caring: } \\
\mathrm{n}^{\circ} \text { of not } \\
\text { re-entered } \\
\text { larvae over } \\
\text { time }\end{array}$ & \begin{tabular}{ccccccc}
\multicolumn{9}{c}{$\mathrm{t}:$} \\
$30 "$ & 1 & 2 & 3 & 4 & 5 & $\mathrm{~min}$ \\
8 & 7 & 5 & 4 & 0 & 0
\end{tabular} & \begin{tabular}{ccccccc}
\multicolumn{9}{c}{$\mathrm{t}:$} \\
$30 "$ & 1 & 2 & 3 & 4 & 5 & \\
10 & 10 & 8 & 8 & 6 & 6
\end{tabular} \\
\hline $\begin{array}{l}\text { Social } \\
\text { interac- } \\
\text { tions: } \mathrm{n}^{\circ} \text { of } \\
\text { aggressive } \\
\text { levels } \\
\text { occurrence }\end{array}$ & \begin{tabular}{lccccc}
\multicolumn{7}{c}{ levels: } \\
0 & 1 & 2 & 3 & 4 & 'a' \\
66 & 38 & 15 & 0 & 0 & 0.14
\end{tabular} & \begin{tabular}{cccccc}
\multicolumn{7}{c}{ levels: } \\
0 & 1 & 2 & 3 & 4 & 'a' \\
27 & 42 & 61 & 0 & 3 & 0.93
\end{tabular} \\
\hline $\begin{array}{c}\text { Cogni- } \\
\text { tion: } \mathrm{n}^{\circ} \\
\text { of ants in } \\
\text { front and } \\
\text { beyond a } \\
\text { difficult } \\
\text { path over } \\
\text { time }\end{array}$ & 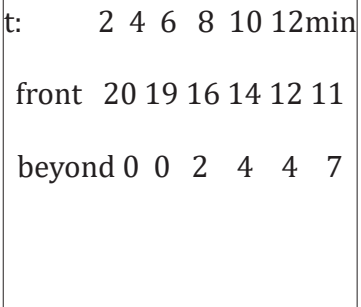 & $\begin{array}{l}\text { t: } \begin{array}{rllllll}2 & 4 & 6 & 8 & 10 & 12 \mathrm{~min} \\
\text { front } & 22 & 20 & 20 & 18 & 16 & 16 \\
\text { beyond } 0 & 0 & 0 & 1 & 2 & 2\end{array}\end{array}$ \\
\hline $\begin{array}{c}\text { Escaping } \\
\text { ability: } \mathrm{n}^{\circ} \\
\text { of escaped } \\
\text { ants over } \\
\text { time }\end{array}$ & t: $\begin{array}{rrrrrr}2 & 4 & 6 & 8 & 10 & 12 \\
2 & 4 & 6 & 9 & 11 & 12\end{array}$ & t: $\begin{array}{lllllll}2 & 4 & 6 & 8 & 10 & 12 & \mathrm{~min} \\
0 & 2 & 4 & 5 & 6 & 7\end{array}$ \\
\hline
\end{tabular}

Table 3: Impact of escitalopram on four ethological and physiological traits.

Details and statistics are given in the text. Briefly, escitalopram affected the ants' social relationships (lines 1,2) and cognition (lines $3,4)$. $t$ = time. Photos of the experiments can be seen in Figure 2. ' $a$ ' $=\mathrm{n}^{\circ}$ levels $2+3+4 / \mathrm{n}^{\circ}$ levels $1+2$.

\section{Brood caring behavior}

This trait was impacted by escitalopram consumption (Table 3, line 1; Figure 2D). Under normal diet, ants promptly found the larvae experimentally removed from the nest, hold them, moved to the nest entrance and re-entered the larvae. While consuming escitalopram, the ants had difficulties in finding the larvae, in holding them, and essentially in walking while holding them. Their legs trembled; they stood still, progressed very slowly and orientated badly towards the nest. Consequently, only few larvae were reentered over the five experimental minutes. The difference of not re-reentered larvae over time between the ants under one and the other kinds of diet was significant: $\mathrm{N}=6, \mathrm{~T}=+21, \mathrm{P}=0.031$. Finally, we had to set the larvae in the nest ourselves. The here observed behavior of ants consuming escitalopram was in agreement with their poor orientation to a tied nestmate, their impacted locomotion, their falling down from the risky tower, their lower percep- 
tion of the rough character of a substrate, four previous made observations (see the four previous subsections relative to these traits). Young ants removed out of the nest together with the larvae appeared to have pronounced difficulties in finding the larvae and essentially in holding them; they finally tried to re-enter their nest without transporting a larva. Their brood caring behavior was thus largely impacted by escitalopram consumption.

\section{Social relationships}

Escitalopram impacted the ants' social interactions (Table 3, line 2; Figure 2E). Under normal diet, two nestmates often stayed side by side doing nothing; they also often contacted each other with their antennae and seldom very slightly opened their mandibles. While consuming escitalopram, two nestmates seldom stayed side by side doing nothing; they often contacted each other with their antennae and often largely opened their mandibles. In the course of the performed dyadic encounters, three flexions of the gaster were observed, what may be a tentative of stinging, i.e. of exhibiting an aggressive behavior. The difference of behavior towards a nestmate between the ants living under normal and the drug diet was significant: $\chi^{2}=45.56, \mathrm{df}=2, \mathrm{P}<0.001$. The ants consuming escitalopram may less perceive the social odor of their nesmates and/or may have a higher level of aggressiveness than when living under normal diet. The here result agreed with that on ants' orientation to a tied nestmate and on ants' brood caring behavior (see the two above subsections which concerns these two traits). No young ant was here tested since they not yet present defense reactions.

\section{Cognition}

Escitalopram affected the ants' ability in crossing a twists and turns path (Table 3, line 3; Figure 3A). Under normal diet, most of the experimented ants entered the twists and turns path and several ones (7 over 12 minutes) could cross it. While consuming escitalopram, few ants entered the difficult path and very few ones ( 2 over 12 minutes) could cross it. The difference of ants' ability in travelling through a twists and turns path between those living under one and the other kinds of diet was significant: in front of the path: $\mathrm{N}=6, \mathrm{~T}=+21, \mathrm{P}=0.016$; beyond it: $\mathrm{N}=4, \mathrm{~T}=-10, \mathrm{P}=0.063$. This may result from the ants' lower activity and audacity while consuming escitalopram (see the subsections relative to these two traits), but may also be caused by an impact of the drug on the ants' cognition. The two following experiments checked this presumption. No young ant was here tested since they not yet present exploratory or foraging behavior.

\section{Escaping capability and state of stress}

Escitalopram affected the ants' escaping capability (Table 3, line 4; Figure 2F). Under normal diet, the ants first moved all around the enclosure area, then calmer along its rim and found so the exit. They easily walked through this notch and went out of the enclosure. After the 12 experimental minutes, the 12 enclosed ants could escape. While consuming escitalopram, the ants also first moved all around the enclosure area, then calmer all along its rim, their state of stress apparently not increasing or decreasing. However, they walked sinuously, trembled, and poorly perceived the exit. Even when duly perceiving the notch, they rarely guessed it could help them escaping, and if finally guessing so, they escaped with difficulty, during more than one minute, or failed to escape. Consequently, the numbers of ants escaped over the 12 experimental minutes were lower than those of ants under normal diet, and this difference was statistically significant $(\mathrm{N}=6, \mathrm{~T}=-21, \mathrm{P}=0.016)$. Such a result was due to the impact of escitalopram on the ants' locomotion and perception, but may also be due to an impact of the drug on the ants' cognition, a presumption the following experiment examined. No young ant was here tested since they do not yet explore and forage.

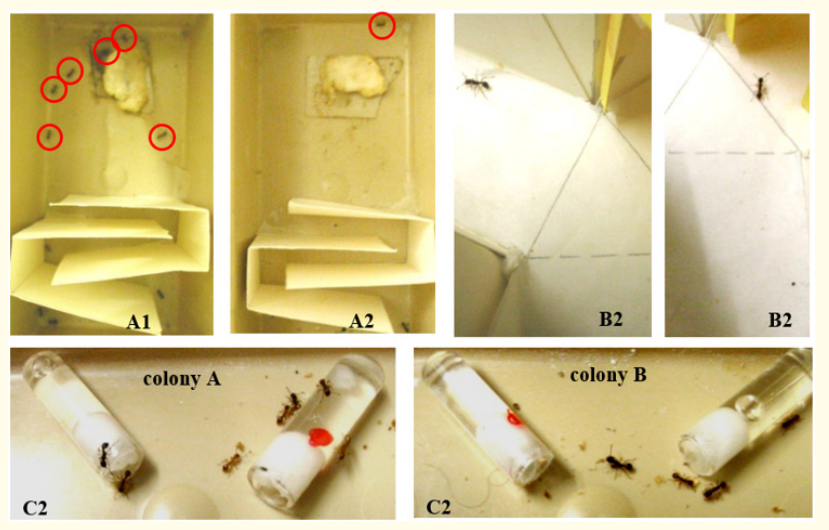

Figure 3: Ant(s) 1 under normal; 2 under escitalopram diet. A: able to cross a twists and turns path while under normal diet, poorly being so while consuming escitalopram. B: trained to a yellow cube, giving the wrong (left photo) and the correct (right photo) when tested in a Y-apparatus. C: in front of a drug-free solution and a solution containing the drug (red dot), not preferentially choosing the latter one, being thus not dependent on escitalopram consumption. 


\begin{tabular}{|c|c|c|}
\hline $\begin{array}{c}\text { Time } \\
\mathbf{h}=\text { hours }\end{array}$ & $\begin{array}{l}\text { Conditioning scores un- } \\
\text { der normal diet }\end{array}$ & $\begin{array}{l}\text { Under a diet with escitalopram } \\
\text { colonies A,B: correct vs wrong responses scores }\end{array}$ \\
\hline $7 \mathrm{~h}$ & $50 \%$ & 5 versus 5,6 versus $455 \%$ \\
\hline $24 \mathrm{~h}$ & $65 \%$ & 4 versus 6,5 versus $545 \%$ \\
\hline $31 \mathrm{~h}$ & $70 \%$ & 5 versus 5,5 versus $550 \%$ \\
\hline $48 \mathrm{~h}$ & $70 \%$ & 4 versus 65 versus $545 \%$ \\
\hline $55 \mathrm{~h}$ & $75 \%$ & 4 versus 65 versus $545 \%$ \\
\hline $72 \mathrm{~h}$ & $80 \%$ & 5 versus 56 versus $455 \%$ \\
\hline $\begin{array}{l}\text { Cue removal } \\
7 \mathrm{~h}\end{array}$ & $70 \%$ & could not be examined \\
\hline $24 \mathrm{~h}$ & $70 \%$ & \\
\hline $31 \mathrm{~h}$ & $70 \%$ & \\
\hline $48 \mathrm{~h}$ & $70 \%$ & \\
\hline $55 \mathrm{~h}$ & $70 \%$ & \\
\hline $72 \mathrm{~h}$ & $70 \%$ & \\
\hline
\end{tabular}

Table 4: Impact of escitalopram on the ants' conditioning ability and memory.

Under esciatalopram, the ants acquired no conditioning. Having learned nothing, their long-term memory could not be examined.

Details are given in the text and photos in figure 2.

\section{Conditioning acquisition, memory}

Escitalopram affected the ants' ability in acquiring conditioning and thus their short- and middle-term memory (Table 4, Figure $3 \mathrm{~B})$. In fact, the ants consuming this drug never acquired conditioning while under normal diet they reached a score of $70 \%$ and $80 \%$ after 31 hours and 72 hours of training respectively. The difference of conditioning acquisition ability between the ants living under one and the other kinds of diet was significant: $\mathrm{N}=6, \mathrm{~T}=+1,-20, \mathrm{P}$ $=0.031$. Since the ants consuming escitalopram acquired no conditioning, we could not study their loss of conditioning, i.e. examine the impact of escitalopram on their long-term memory. However, as these ants went on re-entering their nest and going to the food sites, we can presume that the drug did not affect, at least largely, their long-term memory. No young ant was here tested since they receive their sugar water through trophallaxis from older nestmates and therefore never saw the yellow cube set on the entrance of the tube containing this sugar water.

Ants' adaptation to the impact of escitalopram on their orientation ability

The ants did not adapt themselves to the impact of escitalopram on their orientation ability (Table 5, upper part). After having lived on that drug diet during 8 days, the ants' orientation to a tied nestmate was still significantly lower than that presented under normal

\begin{tabular}{|c|c|c|}
\hline \multicolumn{3}{|c|}{$\begin{array}{c}\text { Ants' orientation to a tied nestmate; median (quartiles) in } \\
\text { angular degrees }\end{array}$} \\
\hline $\begin{array}{c}\text { Under normal } \\
\text { diet }\end{array}$ & $\begin{array}{c}\text { On the drug diet } \\
\text { since } 2 \text { days }\end{array}$ & On the drug diet since 8 days \\
\hline $\begin{array}{c}28.4 \\
(24.8-40.2)\end{array}$ & $\begin{array}{c}62.6 \\
(51.9-81.1)\end{array}$ & $72.9(53.9-97.1)$ \\
\hline
\end{tabular}

Ants' potential dependence on escitalopram consumption

$\mathrm{n}^{\circ}$ of ants on $\quad$ colony A: 23 ; colony B: $17 \rightarrow$ proportion $=$ the drug-free $\quad 54.79 \%$ solution

$n^{\circ}$ of ants on the colony A: 23 ; colony B: $10 \rightarrow$ proportion $=$ drug solution $\quad 45.21 \%$

Upper part: the ants did not adapt themselves to the impact of escitalopram on their orientation ability. After 8 days of the drug consumption, their orientation was still far lower than the control one, and even somewhat lower than that after 2 days of consumption. Lower part: the ants did not develop dependence on the drug consumption. More information is given in the text.

Table 5: Ants' adaptation to the impact of escitalopram on their orientation ability, and ants' dependence on this drug consumption.

$\operatorname{diet}\left(\chi^{2}=38.88, \mathrm{df}=2, \mathrm{P}<0.001\right)$, and was even lower, though not significantly lower, than that presented after 2 days of escitalopram consumption $\left(\chi^{2}=3.13, \mathrm{df}=3,0.20<\mathrm{P}<0.30\right)$. Such an absence of adaptation seemed to also occur for the ants' locomo- 
tion and sensory perception (personal observation). On the basis of the present result, we opted to examine the decrease of the effect of escitalopram after its consumption was stopped using the ants' orientation ability as the trait impacted by the drug (see the subsection relative to this decrease). During the present experiment, no young ant was observed since they not yet respond to the species alarm pheromone [44].

\section{Ants' habituation to a positive effect of escatalopram}

All along our experimental work, we could not point out any positive effect of escitalopram on ants' physiological or ethological traits. Therefore, we could not examine if habituation occurred to the antidepressant effect of escitalopram, but such a habituation may exist for humans and must thus be examined in them since if it occurs, humans will be tempted to increase their consumption of the drug.

\section{Ants' dependence on escitalopram consumption}

The ants did not develop dependence on escitalopram consumption (Table 5, lower part; Figure 3C). Indeed, the tested ants of colony A visited 23 times the solution free of drug and 23 times that containing the drug, while the tested ants of colony B visited 10 times the solution containing the drug and 17 times that free of drug. The proportion of the ants' choice of the drug-free solution equaled thus $54.79 \%$ and that of the ants' choice of the solution containing the drug equaled $45.21 \%$. The numbers of ants sighted in front of each two kinds of solution did not statistically differ from those expected if the ants randomly went towards the two kinds of solution: $\chi^{2}=0.172, \mathrm{df}=1,0.50<\mathrm{P}<0.70$. It could thus be concluded that escitalopram did not induce dependence, at least a physiological one (but humans may develop some psychological dependence on escitalopram). A few young ants were present during this experiment: they did not essentially go to the tube containing escitalopram.

Decrease of the effect of escitalopram after its consumption was stopped

Numerical and statistical results are given in table 6; the decrease is also illustrated in figure 4. Briefly, the effect of escitalopram slowly decreased in a total of about 36 hours. In details, it first stayed unchanged during about 6 hours. Then, until 15 hours after weaning, the effect of the drug decreased but remained statistically not different from its initial one. It became somewhat and significantly lower than its initial effect 18 hours after weaning. After that, it went on decreasing, staying statistically lower than its initial effect and equaling the control value at about 36 hours after weaning. The effect of escitalopram remained highly statistically different from the control situation until 21 after weaning, then it was still though somewhat less significantly different from the control situation until 30 hours after weaning, and finally became not statistically different from the control situation $33-36$ hours after weaning.

Such a decrease accounted for the absence of dependence on the drug consumption (see the subsection relative to dependence). The long lasting effect of escitalopram compared with the control situation (about 36 hours) as well as its significantly lower effect compared with its initial one 18 - 21 hours after weaning is in accordance with the dosage advised to patients, i.e. a dose of $10 \mathrm{mg}$ per day (every 24 hours).

The decrease of the effect of escitalopram after its consumption was stopped appeared to be nearly linear and mathematically described by the function:

$$
E_{t}=E_{i}-1.33 t
$$

With $E_{t}=$ effect at time $t$, in ang. deg.; $E_{i}=$ initial effect, in ang. deg.; $\mathrm{t}=$ time in hours.

To summarize, after a latency period of 6 hours, escitalopram lost about $3 \%$ of its efficiency every hour during about 30 hours.

\section{Discussion and Conclusion}

Antidepressants are among the most consumed drugs in the world, and nowadays, their use is increasing due to the psychological, social and precarious problems generated by the covid-19 pandemic. Escitalopram seems to be preferentially used by practitioners. Even if efficient, being an ISSR, it may have severe side effects. We thus examined its impact on physiological and ethological traits of ants as models and found that this drug affected the food intake, activity, locomotion, orientation, tactile perception, social relationships, cognition, learning, as well as short and middle-term memory. No adaptation occurred to these side effects, and the ants did not develop dependence on escitalopram consumption. After weaning, the effect of escitalopram stayed intact for 6 hours, then lost 3\% every hour, becoming different from its initial one 18 hours after weaning and similar to the control situation after a total of about 36 hours. We observed (without quantifying it) that the young ants were more affected than the old ones by escitalopram consumption. Here below, we comment our results, compare them with those of other researchers, and conclude. 


\begin{tabular}{|c|c|c|c|c|c|c|c|}
\hline \multirow{2}{*}{$\begin{array}{c}\text { Time } \\
\text { h = hours }\end{array}$} & \multirow{2}{*}{$\begin{array}{c}\text { Orientation } \\
\text { ang.deg. }\end{array}$} & \multicolumn{3}{|c|}{ statistics versus $\mathrm{t}=0$} & \multicolumn{3}{|c|}{ statistics versus control } \\
\hline & & $\chi^{2}$ & df & $\mathbf{P}$ & $\chi^{2}$ & df & $\mathbf{P}$ \\
\hline Oh & $71.5(63.6-80.3)$ & & & & & & \\
\hline $3 \mathrm{~h}$ & $73.7(61.7-80.7)$ & 0.52 & 2 & $<0.80$ & 27.84 & 2 & $<0.001$ \\
\hline $6 \mathrm{~h}$ & $71.9(57.6-86.4)$ & 1.80 & 1 & $<0.50$ & 33.75 & 2 & $<0.001$ \\
\hline $9 \mathrm{~h}$ & $63.7(53.2-83.2)$ & 0.47 & 1 & $\sim 0.50$ & 34.60 & 2 & $<0.001$ \\
\hline $12 \mathrm{~h}$ & $60.0(50.2-67.6)$ & 0.63 & 1 & $<0.50$ & 24.49 & 2 & $<0.001$ \\
\hline $15 \mathrm{~h}$ & $58.6(49.0-69.8)$ & 0.63 & 1 & $<0.50$ & 24.49 & 2 & $<0.001$ \\
\hline $18 \mathrm{~h}$ & $54.0(46.4-68.5)$ & 5.23 & 1 & $<0.05$ & 18.53 & 2 & $<0.001$ \\
\hline $21 \mathrm{~h}$ & $52.4(46.0-60.9)$ & 12.13 & 1 & $<0.001$ & 16.27 & 1 & $<0.001$ \\
\hline $24 \mathrm{~h}$ & $47.7(35.1-60.4)$ & 12.13 & 1 & $<0.001$ & 5.27 & 1 & $<0.05$ \\
\hline $27 \mathrm{~h}$ & $47.7(35.6-53.4)$ & 14.4 & 1 & $<0.001$ & 8.91 & 1 & $<0.01$ \\
\hline $30 \mathrm{~h}$ & $41.8(35.3-51.9)$ & 14.4 & 1 & $<0.001$ & 8.91 & 1 & $<0.01$ \\
\hline $33 \mathrm{~h}$ & $33.9(30.3-47.6)$ & 8.28 & 11 & $<0.001$ & 0.62 & 1 & $<0.50$ \\
\hline $\begin{array}{c}\text { 36h } \\
\text { Control }\end{array}$ & $\begin{array}{l}31.5(23.4-44.0) \\
28.4(24.8-40.2)\end{array}$ & 19.80 & & $<0.001$ & 0.64 & 1 & $<0.50$ \\
\hline
\end{tabular}

Table 6: Decrease of the effect of escitalopram after its consumption was stopped.

After weaning, the effect of escitalopram remained intact for 6 hours, then slowly decreased, becoming different form its initial effect 18 hours after weaning and similar to the control situation about 36 hours after weaning. This kind of decrease was in agreement with the absence of dependence on the drug consumption.

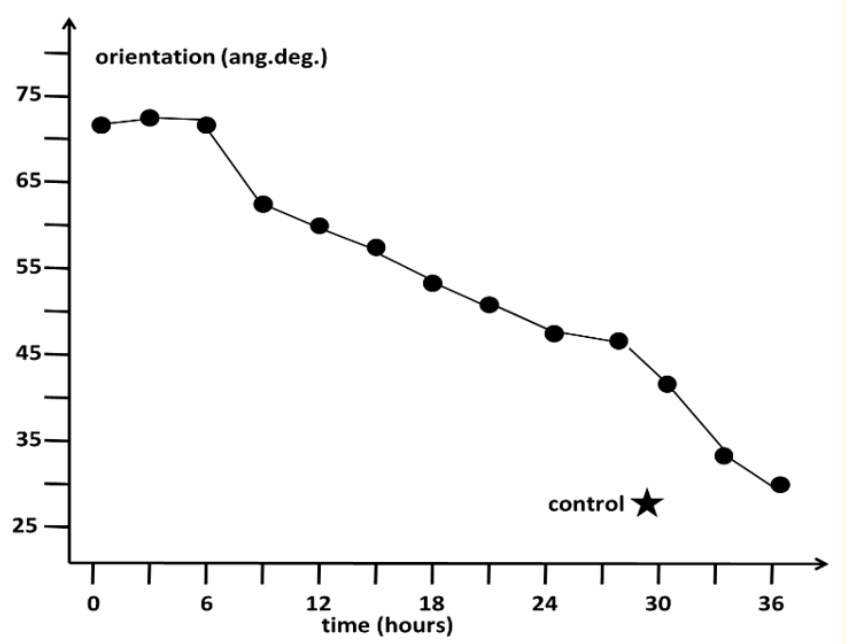

Figure 4: After a latency period of 6 hours, the effect of escitalopram slowly, nearly linearly decreased, losing about $3 \%$ of its value every hour, becoming significantly different from its initial value 18 hours after weaning and significantly similar to the control value after a total of about 36 hours. Details are given in the text, numerical and statistical results in table 6.
The results obtained for the different physiological and ethological examined traits were in agreement with each other, among others those on activity, audacity and locomotion, on orientation and tactile perception, on brood caring, locomotion, orientation, tactile perception and social relationships, on cognition, escaping ability and conditioning acquisition, as well as on the absence of dependence and the slow decrease of the effect of escitalopram after weaning. Most of the pointed out effects of escitalopram on ants correspond to those reported for humans (see below). Ants appeared thus to be excellent biological models for such kind of study. A few side effects not (or not yet) observed in humans have been seen in ants and might thus occur in humans, e.g. effect on tactile perception, social relationships, cognition, learning, shortand middle-term memory, as well as no adaptation to side effects. In ants, habituation to a positive effect of escitalopram could not be examined. This should be done in humans since, if habituation occurs, humans will be tempted to increase their drug consumption and consequently the development of side effects.

Concerning the studies made in humans or in other animals as models, first, escitalopram seems to be an efficient antidepressant 
when compared with a placebo and even with other antidepressants. The efficient dose is meanly $10 \mathrm{mg}$ per day, independently of the patients' weight and poorly of their age [45-47].

As for the harmful side effects described in humans and partly reported in the introduction section, escitalopram induces fewer side effects than the other antidepressants because, compared to them, it lacks affinity for postsynaptic receptors what contributes to side effects [19]. However, several clinical studies on the side effects attributed to citalopram and escitalopram affirmed that these drugs lead to difficulty returning to sleep after awakening, nausea, tremors, and drowsiness among others [see references in the introduction section]. These effects which occur in humans are in agreement with several ones observed in ants, such as decrease of food intake, activity, audacity and escaping ability. A clinical trial revealed that escitalopram can relieve pain in patients with painful polyneuropathy, but that this drug cannot nevertheless be as a standard treatment in neuropathic pain: $10.4 \%$ of the patients discontinued the use of escitalopram due to its side effects [48]. The above effect of escitalopram on humans' pain perception is in agreement with our finding of the effect of that drug on the ants' tactile (pain) perception. In the notice joined to escitalopram packages, aggressiveness is reported as a potential side effect; we effectively observed some aggressive behavior in ants consuming this drug. A special study of the side effects of citalopram and escitalopram has been made for the Canadian Air Forces and has shown that these drugs did not affect the patients' reaction time, logical reasoning, serial subtraction ability and multitasks performance, and could thus be used 'under close observation' [49]. This study may not have been made without any conflict of interest (see below). We have already cited in the introduction section the detailed analysis, made on 211 patients during 12 weeks, of the side effects of escitalopram and duloxetine, including frequencies, time to onset, duration, dose responsiveness, and impact on treatment outcomes [20]. The results of this study should help prescribers making their medication decisions being well informed. Even in the latter detailed mentioned study, nothing is reported about the impact on humans' relationships, cognition, learning, memory, adaptation to side effects, habituation to positive effects, dependence of escitalopram consumption, and how the effect of the drug decreases after weaning. Our present work on ants examined such impacts except the potential habituation to positive effects of the drug, what should thus be made on humans. Concerning the other here examined side effects, we finally found two works showing that escitalopram may shorten humans' attention, and may induce children's behavioral disinhibition $[50,51]$. The results of the two latter cited studies are in agreement with our finding of some impact of escitalopram on ants' cognition, learning, and maybe relationships. They seem being not in accordance with the conclusion of a here above cited study made for the Canadian Air Forces [49].

To conclude, escitalopram appears to be efficient as an antidepressant and to be generally more efficient and safer than other similar drugs. However, it still has side harmful effects we clearly pointed out on ants. Most of them are in agreement with those observed in humans; a few ones have not yet been described in humans and should thus be examined in them. Also, it is of interest to check if humans acquire some habituation to positive effects of escitalopram since if this occurs, they will be tempted to increase their drug consumption. Let us add that ants appeared to be excellent biological models, as they were in the course of our previous investigations on the effects of products used by humans [52].

\section{Ethical Considerations, Conflict of Interest}

We affirm having maintained the ants in the best possible environmental condition. We also declare having no conflict of interest as for the pharmacists', practitioners' and humans' use of escitalopram or of any other similar (competitive) drugs. We work on ants, on their biology, cognition, numerosity ability, and ontogenesis of their capabilities, and we receive no money for making our research.

\section{Bibliography}

1. www.santemagazine.fr > Actualités > Actualités traitement

2. Health NIoM. "Prevalence of Major Depressive Episode Among Adults" (2018).

3. Cammaerts MC., et al. "Physiological and ethological effects of fluoxetine, on ants used as biological models" International Journal of Biology 7.2 (2015): 1-18.

4. Cammaerts MC., et al. "Ethological and physiological effects of paroxetine, the most consumed antidepressant nowadays: a study on ants as models". Trends in Entomology 12 (2016): 107-125.

5. Cammaerts MC., et al. "Physiological and ethological effects of antidepressants: a study using ants as biological models". International Journal of Pharmaceutical Science Invention 4.2 (2015): 4-24. 
6. Cammaerts MC., et al. "Amitriptyline: physiological and ethological effects of a largely used antidepressant evaluated on ants as biological models". Acta Scientific Pharmaceutical Sciences.

7. Bondolfi G., et al. «Psychiatrie: l'escitalopram (Cipralex $\left.{ }^{\circledR}\right) »$. Revue Médicale Suisse 1 (2003): 22620.

8. Sanchez C., et al. "The S-enantionner of citalopram (Lu 26-054) is a highly selective and potent serotonin reuptake inhibitor". Biological Psychiatry 47 (2000): 291.

9. Owens MJ., et al. "Second-generation SSRIs: Human monoamine transporter binding profile of escitalopram and R-fluoxetine". Biological Psychiatry 50.5 (2001): 345-350.

10. Gorman JM., et al. "Efficacy comparison of escitalopram and citalopram in the treatment of major depressive disorder: Pooled analysis of placebo-controlled trials". CNS Spectrum 7 (2002): 40-44.

11. Kennedy SH., et al. "Efficacy of escitalopram in the treatment of major depressive disorder compared with conventional selective serotonin reuptake inhibitors and venlafaxine XR: a meta-analysis". Journal of Psychiatry and Neuroscience 31.2 (2006): 122-131.

12. Burke WJ., et al. "Fixed-dose trial of the single isomer SSRI escitalopram in depressed outpatients" Journal of Clinical Psychiatry 63 (2002): 331-336.

13. Wades A., et al. "Escitalopram $10 \mathrm{mg} /$ day is effective and well tolerated in a placebo-controlled study in depression in primlary care". International Journal of Clinical Psychopharmacology 17.3 (2001): 95-102.

14. Lepola UM., et al. "Escitalopram (10-20mg/DAY) is effective and well tolerated in a placebo controlled study in depression in primary care". International Journal of Clinical Psychopharmacology 18.4 (2003): 211-217.

15. Millet B. "Thérapeutique. Escitalopram versus IRSNa". L'Encéphale 34.3 (2008): 280-283.

16. Robinson RG., et al. «Escitalopram et psychothérapie de résolution des problèmes dans la prévention des dépressions après accident vasculaire cérébral». JAMA Article Clinique Original 299.20 (2008): 2391-2400.
17. Bélicard C., et al. "Antidepressant use in demented elderly subjects: current data". La Revue de Médecine Interne 30.11 (2009): 947-954.

18. Fish EW., et al. "Anxiolytic-like effects of escitalopram, citalopram, and R-citalopram in maternally separated mouse pups". Journal of Pharmacology and Experimental Therapeutics 308.2 (2004): 474-480.

19. Burke WJ. "Escitalopram". Expert Opinion on Investigational Drugs 11.10 (2002): 1477-1486.

20. Polychroniou PE., et al. "Temporal profiles and dose-responsiveness of side effects with escitalopram and duloxetine in treatment-naïve depressed adult". Behavioral Sciences 8.7 (2018): 64.

21. Cammaerts MC. "Harmful effects of humans' environmental factors and drugs, and advices for a safer live; a study on ants as models". World Journal of Pharmaceutical Science 9.1 (2021): 34-45.

22. Wehner R., et al. «Biologie et physiologie animales». Eds. De Boeck Université, Thieme Verlag, Paris, Bruxelles (1999).

23. Russell WMS., et al. "The Principles of Humane Experimental Technique". Johns Hopkins University (2014).

24. Wolf FW., et al. "Invertebrate models of drug abuse". Journal of Neurobiology 54 (2003): 161-178.

25. Andre RG., et al. "Insect Models for Biomedical Research". In: Nonmammalian Animal Models for Biomedical Research. Woodhead AD, editor, Boca Raton, FL: CRC Press (1989).

26. Passera L., et al. «Les fourmis: comportement, organisation sociale et évolution». Les Presses Scientifiques du CNRC, Ottawa, Canada (2005).

27. Cammaerts MC., et al. "Comparative outlook over three Myrmica species' biotopes and foragers' know-how" Biologia 69 (2014): 1051-1058.

28. Cammaerts MC., et al. "Ontogenesis of ants' cognitive abilities (Hymenoptera, Formicidae)". Advance Study in Biology 7 (2015): 335-348 + synopsis: 349-350.

29. Cammaerts MC., et al. "Are ants (Hymenoptera, Formicidae) capable of self recognition?". Journal of Sciences 5.7 (2015): 521-532. 
30. Cammaerts MC., et al. "Ants'numerosity ability defined in nine studies". Journal of Biology and Life Science 11.1 (2020): 121142.

31. Cammaerts MC., et al. "Summary of seven more studies on numerosity abilities in an ant, four of them relating to human competence" Journal of Biology and Life Science 11.2 (2020): 296-326.

32. Cammaerts MC., et al. "Non-numerical distance and size effects in an ant". Journal of Biology and Life Science 11.2 (2020): 13-35.

33. Cammaerts MC., et al. "Weber's law applied to the ants' visual perception". Journal of Biology and Life Science 11.2 (2020): 36-61.

34. Cammaerts MC., et al. "Ants can expect the time of an event on basis of previous experiences". Entomology (2016): 9.

35. Cammaerts MC., et al. "Spatial expectation of food location in an ant on basis of previous food locations (Hymenoptera, Formicidae)". Journal of Ethology 35.1 (2016): 9.

36. Cammaerts MC., et al. "Ants anticipate the following quantity in an arithmetic sequence". Behavioral Sciences 11.2 (2021): 13.

37. Cammaerts MC. "Can Myrmica rubra ants use tools or learn to use them?". International Journal of Biology 10.1 (2018): 1-12.

38. Cammaerts MC. "Is the largely used analgesic paracetamol without any adverse effects? A study on ants as models". EC Pharmacology and Toxicology 4.2 (2017): 51-68.

39. Cammaerts MC., et al. "Ants as models for assessing the effects on health of $\mathrm{CaCO}_{3}+\mathrm{MgCO}_{3}$ mixture used to decrease gastric hyperacidity" EC Nutrition 13.7 (2018): 500-514.

40. Cammaerts MC., et al. "Side effects of chloroquine and hydrochloroquine examined on ants as models". EC Pharmacology and Toxicology 8.11 (2020): 57-82.

41. Siegel S and Castellan NJ. «Nonparametric statistics for the behavioural sciences". Singapore: McGraw-Hill (1998).

42. Cammaerts MC., et al. "An easy and cheap software-based method to assess two-dimensional trajectories parameters". Belgian Journal of Zoololgy 142 (2012): 145-151.
43. Cammaerts MC., et al. "Ethological and physiological effects of Sativex, a cannabis-based medicine, examined on ants as models". Acta Scientific Pharmaceutical Sciences 4.8 (2020): 63-84.

44. Cammaerts MC. "Performance of the species-typical alarm response in young workers of the ant Myrmica sabuleti is induced by interactions with mature workers". Journal of Insect Science 14.1 (2014): 234.

45. Spadone C. "Severe forms of depression: the efficacy of escitalopram”. L'Encéphale 35.2 (2009): 152-159.

46. Montgomery SA., et al. "Escitalopram (S-Enantiomer of Citalopram): Clinical Efficacy and Onset of Action Predicted from a Rat Model". Pharmacology and Toxicology 88.5 (2001): 282 286.

47. Owley T., et al. "An open-label trial of escitalopram in pervasive developmental disorders". Journal of the American Academy of Child and Adolescent Psychiatry 44.4 (2005): 343-438.

48. Otto M., et al. "Escitalopram in painful polyneuropathy: a randomized, placebo-controlled, cross-over trial". Pain 139.2 (2008): 275-283.

49. Paul MA., et al. "SSRI effects on psychomotor performance: assessment of citalopram and escitalopram on normal subjects". Aviation, Space, and Environmental Medicine 78.7 (2007): 693607.

50. Drucke B., et al. "Differential effects of escitalopram on attention: a placebo-controlled, double-blind cross-overstudy". Psychopharmacology 207.213 (2009).

51. Coskun M., et al. "Escitalopram treatment in preschool children with anxiety disorders: a case series". Bulletin of Clinical Psychopharmacology 22.3 (2012): 262-267.

52. Cammaerts MC. "Harmful effects of humans' environmental factors and drugs, and advices for a safer live; a study on ants as models". World Journal of Pharmaceutical Science 9.1 (2021): 34-45.

Volume 5 Issue 6 June 2021

(C) All rights are reserved by Marie-Claire Cammaerts. 